\title{
Complicated Sigmoid Diverticulitis Presenting as Severe Necrotizing Soft Tissue Infection
}

\author{
Mahmoud H. Alghamdi, Rachel G. Khadaroo, ${ }^{1,2}$ and Kamran Fathimani ${ }^{1}$
}

\begin{abstract}
Background: Necrotizing soft tissue infection is a rare and aggressive medical and surgical emergency characterized by intense and severe tissue destruction, rapid onset systemic toxicity, and high mortality. Diverticulitis is a common complication of diverticular disease. However, its presentation as necrotizing soft tissue infection is rare. It is always important to identify the precipitating cause of necrotizing soft tissue infection to assist in appropriate treatment and risk stratification.

Case Presentation: The patient was an 84-year-old female with a history of hypertension and chronic obstructive pulmonary disease with long-term steroid dependency. She presented to the emergency department with abdominal tenderness, left abdominal wall cellulitis, crepitus, and septic shock. Abdominal and pelvic computed tomography findings showed intra-abdominal abscess secondary to perforated sigmoid diverticulitis with abdominal wall abscess and necrotizing soft tissue infection of abdominal wall, chest wall, groin, and back. After intravenous fluid resuscitation and antibiotics, the patient underwent urgent laparotomy. She was found to have a large abdominal wall abscess, perforated sigmoid diverticulitis, and extensive necrosis of the torso extending from the groin to chest wall including the back.

Conclusion: Abdominal wall necrotizing soft tissue infection is an uncommon complication of perforated diverticulitis. In an elderly immunocompromised patient with septic shock, perforated diverticulitis, and necrotizing soft tissue infection, the prognosis is poor.
\end{abstract}

Keywords: diverticulitis; necrotizing soft tissue infection; subcutaneous emphysema; corticosteroid; septic shock

$\mathbf{N}$ ECROTIZING SOFT TISSUE INFECTION (NSTI) is defined by necrosis of subcutaneous tissue, fascia, and muscle, which could be lethal with estimated mortality rate of $23.5 \%$ [1] if not treated promptly with proper antibiotics and surgical debridement. Polymicrobial infections are the most common form of NSTI [2]. This occurs rarely secondary to gastrointestinal tract perforations such as from carcinoma or diverticulitis [2]. We present a case of complicated sigmoid diverticulitis with abdominal wall abscess and extensive abdominal wall, groin, flank, back, and chest wall NSTI in an elderly patient.

\section{Case Presentation}

The 84-year-old female had a past medical history of chronic obstructive pulmonary disease (COPD) with long- term steroid use and hypertension. She developed abdominal tenderness, elevated temperature, tachycardia, and hypotension. On physical examination, there was left abdominal wall cellulitis with crepitus consistent with an NSTI. The patient's white blood cell count was normal. To understand what caused this NSTI a computed tomography (CT) scan of the abdomen and pelvis was performed revealing sigmoid diverticulitis complicated by a pericolonic abscess and left anterior abdominal wall abscess (Fig. 1). CT scan also showed the extent of the NSTI, which involved the left abdominal wall, flank, back, and chest (Fig. 2). The patient was diagnosed with septic shock caused by sigmoid diverticulitis complicated by abdominal wall abscess and NSTI. She was classified as a high-risk patient given her age and severity of septic shock. Surgery

\footnotetext{
${ }^{1}$ Department of Surgery, ${ }^{2}$ Department of Critical Care Medicine, Faculty of Medicine and Dentistry, University of Alberta, Edmonton, Alberta, Canada.

(C) Mahmoud H. Alghamdi et al. 2017; Published by Mary Ann Liebert, Inc. This Open Access article is distributed under the terms of the Creative Commons License (http://creativecommons.org/licenses/by/4.0), which permits unrestricted use, distribution, and reproduction in any medium, provided the original work is properly credited.
} 


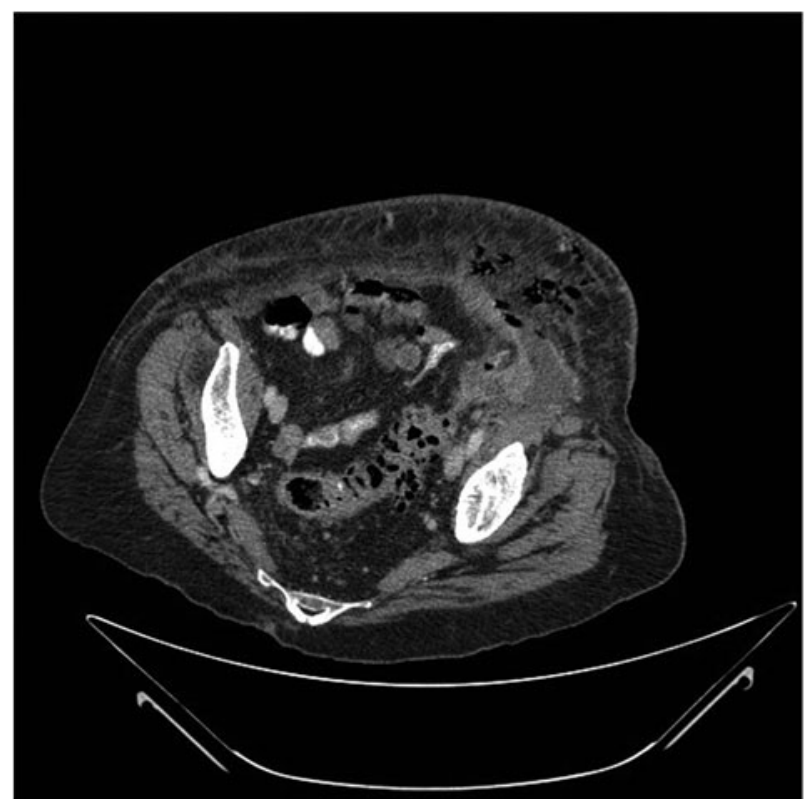

FIG. 1. Sigmoid diverticulitis complicated by peri-colonic abscess and abdominal wall subcutaneous emphysema.

was performed and a large left lower abdominal wall abscess was drained. Laparotomy showed multiple left colonic diverticula consistent with diverticulitis. There was a large amount of sigmoid inflammation with an extension to the upper rectum. The inflammatory mass was not resectable because of the patient's intra-operative instability. There was extensive left abdominal wall necrosis and an NSTI involving the entire abdominal wall musculature with extension to the left flank, back, groin, and left lower chest

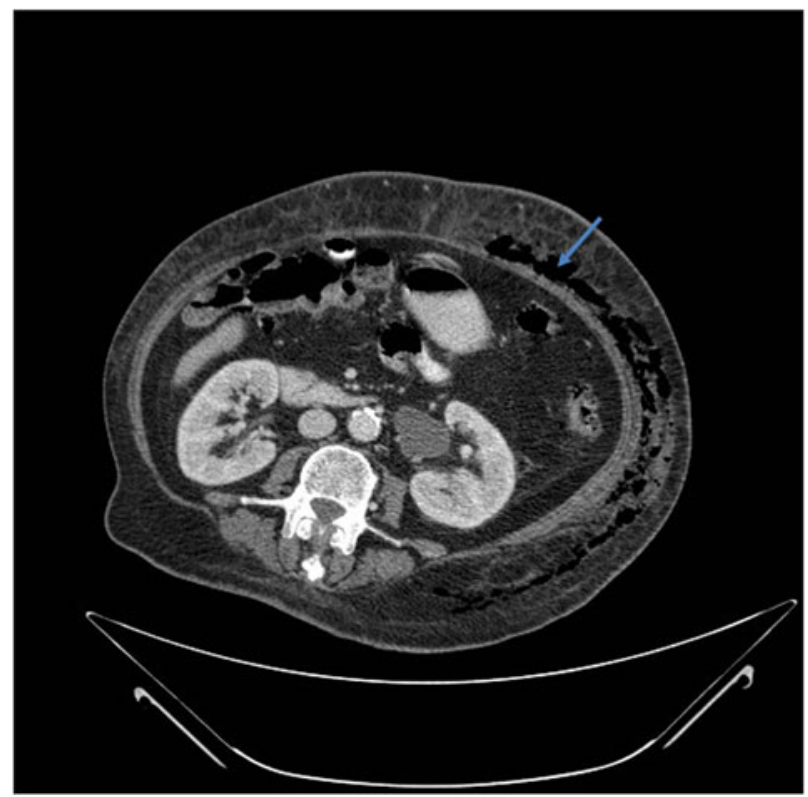

FIG. 2. Subcutaneous emphysema of left abdominal wall (arrow), flank, and back in a patient with sigmoid diverticulitis. wall. Surgical debridement of the left rectus abdominis muscle, external oblique aponeurosis, latissimus dorsi muscle, and left flank and back abdominal musculature was performed. This resulted in a significant defect of the anterior abdominal wall. The midline abdominal fascia could not be closed because only the left posterior sheath of the rectus abdominis muscle remained. A diverting colostomy was also deferred at this time because the patient was in septic shock with dependence on vasopressors throughout the entire procedure. Saline-soaked dressings were placed on top of the remaining abdominal wall. As a result of significant loss of abdominal wall domain and septic shock, the prognosis was anticipated to be poor with a nonsurvivable clinical situation. Given the aggressive nature of the NSTI, a decision was made with the family for compassionate care. The patient died within hours after the operation.

\section{Discussion}

The mortality rate of NSTI is high. In 67 studies done in the period between 1980 and 2008, a mortality rate of $23.5 \%$ has been reported [3]. Independent predictors of mortality for NSTI have been identified including: age older than 75 years, severe peripheral vascular disease, hospital-acquired NSTI, multifocal NSTI, and septic shock at admission [1]. In immunocompromised patients, such as long-term corticosteroid use, the post-operative mortality rate for diverticulitis surgery can be as high as $31.6 \%$ [4]. Lack of early symptoms in the disease presentation hinders timely diagnosis and treatment [5-7].

Necrotizing soft tissue infection is a surgical emergency and the diagnosis is often made clinically without the need of imaging tools. However, in the cases in which a primary source of infection is suspected, to assist in more rapid diagnosis, or those in which the extent of disease may impact clinical decision, imaging can be an important modality. It is important to remember that the CT can be have negative or non-specific findings and cannot rule out NSTI especially early in the disease process [8].

In this case, despite aggressive surgical debridement, the patient had three of the five major risk factors for death: advanced age, extensive NSTI with a major intra-abdominal primary source of infection, and septic shock. This in combination with her immunosuppressed status resulted in a poor outcome.

\section{Conclusion}

Computed tomography can be a useful modality to diagnosis intra-abdominal sources of NSTI. Determining severity of NSTI on admission and knowledge of patient risk factors can predict the likelihood of mortality. This can assist in discussions with patients and families.

\section{Acknowledgments}

No funding sources or assistance were necessary for production of this manuscript.

\section{Author Disclosure Statement}

No competing financial interests exist. 


\section{References}

1. Hua C, Sbidian E, Hemery F, et al. Prognostic factors in necrotizing soft-tissue infections (NSTI): A cohort study. J Am Acad Dermatol 2015;73:1006-12.e8.

2. Sarani B, Strong M, Pascual J, et al. Necrotizing fasciitis: Current concepts and review of the literature. J Am Coll Surg 2009;208:279-288.

3. May AK. Skin and soft tissue infections. Surg Clin North Am 2009;89:403-420.

4. Biondo S, Trenti L, Elvira J, et al. Outcomes of colonic diverticulitis according to the reason of immunosuppression. Am J Surg 2016;212:384-390.

5. Wong C, Chang H, Pasupathy S, et al. Necrotizing fasciitis: Clinical presentation, microbiology, and determinants of mortality. J Bone Joint Surg Am 2003;85-A: 1454-1460.

6. Puvanendran R, Huey JCM, Pasupathy S. Necrotizing fasciitis. Can Fam Physician 2009;55:981-987.

7. Kalaivani V, Hiremath BV, Indumathi VA. Necrotising soft tissue infection-risk factors for mortality. J Clin Diagn Res 2013;7:1662-1665.

8. Chaudhry AA, Baker KS, Gould ES, et al. Necrotizing fasciitis and its mimics: What radiologists need to know. AJR Am J Roentgenol 2015;204:128-139.
Address correspondence to:

Dr. Rachel G. Khadaroo

Faculty of Medicine and Dentistry

Department of Surgery

University of Alberta

2D3 Walter C. Mackenzie Health Sciences Centre 8440112 Street NW

Edmonton, Alberta, T6G $2 R 7$

Canada

E-mail: khadaroo@ualberta.ca

Abbreviations Used
$\mathrm{COPD}=$ chronic obstructive pulmonary disease
$\mathrm{CT}=$ computed tomography
$\mathrm{NSTI}=$ necrotizing soft tissue infection

Cite this article as: Alghamdi $\mathrm{MH}$, Khadaroo $\mathrm{RG}$, Fathimani K (2017) Complicated sigmoid diverticulitis presenting as severe necrotizing soft tissue infection. Surgical Infections Case Reports 2:1, 14-16, DOI: 10.1089/crsi.2016.0057 